\title{
Towards an autonomous RIRS: design, structure investigation and framework
}

\author{
Miftahur Rahman \\ School of Aerospace, Transport \\ and Manufacturing \\ Cranfield University \\ Cranfield, United Kingdom \\ miftahur.rahman@cranfield.ac.uk
}

\author{
Haochen Liu \\ School of Aerospace, Transport \\ and Manufacturing \\ Cranfield University \\ Cranfield, United Kingdom \\ haochen.liu@cranfield.ac.uk
}

\author{
Isidro Durazo Cardenas \\ School of Aerospace, Transport \\ and Manufacturing \\ Cranfield University \\ Cranfield, United Kingdom \\ i.s.durazocardenas@cranfield.ac.
}

uk

\author{
Amanda Hall \\ Technical Authority \\ Network Rail \\ United Kingdom \\ amanda.hall@networkrail.co.uk
}

Robert Anderson

Technical Services Network Rail

Milton Keynes, United Kingdom robert.anderson4@networkrail.co

\begin{abstract}
Automated robots have been deeply embedded in many industries for decades. Autonomous railway maintenance is attracting more attention, but few robotic technologies are used in rolling stock inspection and repair. Due to geometrical differences between rail and road, wheeled robots still need to fulfill research and deployment gaps for application in railway track maintenance. This research is intended to design an autonomous Robotic Inspection and Repair System (RIRS) for unmanned track maintenance. It mainly employs commercial Warthog Unmanned Ground Vehicle (UGV), Universal Robot (e.g., UR10e) manipulator, and multiple onboard sensors to achieve navigation to track, road-rail conversion, and on-track inspection and repair. With the support of a trolley, RIRS will achieve the ability to operate both on-track and off-track. This research also investigates the system structure of the on-track inspection and repair by considering the dynamic degree-of-freedom of both UGV wheels and the joints of robot manipulator. The redundancy of joints for the mobile manipulator has been reduced by proposing simplified joints which will improve the performance and efficiency. This research analyses the dynamic principles of a new maintenance system that will be deployed and tested in a prototype RIRS system in future work.
\end{abstract}

Keywords - Road Rail Vehicle, Rail Inspection and Repair System, UGV, manipulator, autonomous

\section{INTRODUCTION}

The railway is a widely used mass transport medium which has been serving people and carrying goods for several hundreds of years. It is expected that the use of the railway will increase in the upcoming years. For example, in the UK, the number of passengers in trains will be doubled and freights will rise by $90 \%$ in 2041 compared to 2014[1]. This enormous increase in demand can only be fulfilled by ensuring less track downtime and ensuring proper inspection and repair work. Most of the inspection and repair work is carried out by handheld machines driven manually by a human or specially equipped trains. Maintenance done by a human with a handheld device is slow .uk

and costly while specially equipped trains such NMT (New Measurement Train) [2], Doctor Yellow [3], China Railway Comprehensive Inspection Trains [4], etc. has only the capability to identify rail defects but requires human for onboard monitoring and driving the train.

The financial advantage of robotics automation in rolling stock maintenance and related tasks has been already proven [5], [6]. A miniature robot model have been used to demonstrate fault detection [7] and cleaning the track [8], [9] or performing repair job such as 3D printing [10]. Safe human-robot interactions in railway maintenance task has been demonstrated in [11]. A combination of wheeled robot and a manipulator is named as a mobile manipulator and can perform many risky and remote operational task such as tunnel inspection [12] or cleaning of the nuclear reactor [13]. The mobile manipulator has great potential to be used in railway track inspection and repair and this potential use was visualized in simulation environment [14]. The only drawback is that it will suffer while moving along the track and may get stuck in the crossings with the conventional pneumatic tyre. Replacing the pneumatic tyre with rail wheel-shaped tyre may solve the problem of moving ontrack but it will lose the capability of running off-track which reduces the versatility. Converting the mobile manipulator into a RRV (Road Rail Vehicle) can be a promising solution. There are few ready to use RRV available commercially from Permaquip and Aquarius. But they are fuel-driven and uses traditional drive and steering system which will require a lot of modification to achieve autonomous operation. AutoScan, an autonomous rail inspection trolley with scanners, can detect the defects in the track. But, the system is only capable of inspecting the track [15]. Trolley and robotic arm equipped with laser and ACFM scanner was proposed in [16] which can only be used for on track inspection. Railpod can run both on the track and off the track, as it has both reconfigurable mechanisms to switch between rail wheel and pneumatic wheel [17]. Without the arm, 
it is unable to further inspect the crack and the pneumatic wheel mechanism is only capable of running on a plain surface.

\section{MOTIVATION}

Defects on the rail pose a safety risk for the passenger and train. Vegetation near the track or unwanted material lying near or on the track may also pose a serious threat to the safety of the passenger and train. Most of the researchers are focusing only on on-track inspection or repair, while a complete autonomous RIRS should have the capability to conduct both on-track maintenance and off-track operation (such as unmanned travel from base to track, road-rail transformation, etc.). IN2SMART2 (Intelligent Innovative Smart Maintenance of Assets by integRated Technologies 2) is a project under Shift2Rail program to improve the Technology Readiness Level (TRL) for the RIRS command and control system. This research is aimed to give a comprehensive and effective solution that will convert a mobile manipulator into a RIRS which can be used in the future for both on-track and off-track operation. A command and control system for an autonomous RIRS will be developed and tested to check the TRL of autonomous RIRS.

\section{PROPOSED RIRS SOLUTION}

An autonomous ground vehicle, a type of wheeled robots, such as Warthog from Clearpath Robotics, can run in all types of terrain. Manipulators such as UR10e arm which has 6 degrees of freedoms from Universal Robotics has been used in many industrial applications. The proposed RIRS consists of a mobile manipulator and a trolley which will perform two tasks: inspection and repair. In the inspection task, it will inspect the track while travelling with an arm head camera and detect defects and save the exact defects location for future reference. In the repair task, it will use the manipulator and gripper to perform proper repair job according to defect type. In addition, the Road Rail Conversion will be implemented to fulfill both the on-track and off-track operation requirements and flexible transformation for supporting train schedules.

\section{A. Higher level task completion framework}

For achieving autonomy, RIRS needs to interface with a higher-level central control centre (CCC) and exchange data such as task command: RIRS status and location, train signal, track availability for repair, inspection and repair data, etc. The system is designed to perform both inspection and repair tasks both on on-track and off-track operation mode. For changing the operation mode, RRC (Road Rail Conversion) will take place. Higher level task completion framework is shown in Figure 1.

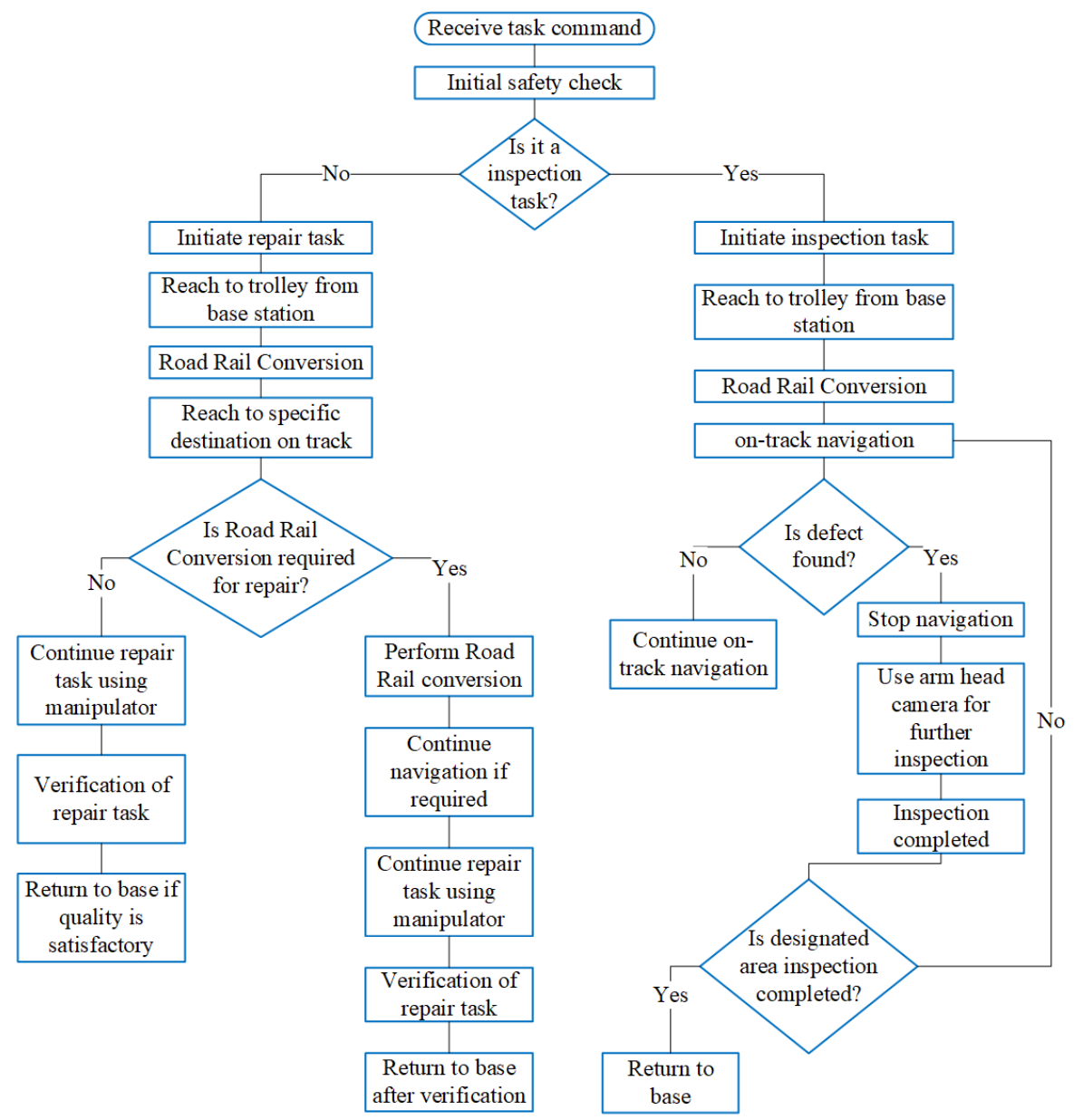

Fig. 1. Higher level task completion framework for autonomous RIRS 


\section{B. Hardware}

The proposed solution is comprised of three major components. The first one is Warthog UGV from Clearpath Robotics which can run in most terrain at a maximum speed of $18 \mathrm{~km} / \mathrm{hr}$, have a skid steering drive system, and have onboard computation device which has ROS and MATLAB API. The second component is the manipulator supplied with the UGV, from Universal Robotics. It has six degrees of freedom and has arm head camera and two-finger end-effector from Robotiq. The arm head camera can be used for subtle defect inspection with improved localization for future reference and the endeffector can be used for repair task. All these are readily available for commercial use. Third and last major component is a trolley which provides on-rail conversion. It has four 10inch rail wheels (10inch rail wheel) and 8 contact rollers. This trolley will guide the mobile manipulator along the railway track. Though the trolley has no power source of its own, UGV will control the movement of this trolley. Power will be transmitted in 2 stages; first, the pneumatic wheel of warthog will rotate the contact rollers with the help of friction and the power will be transmitted to the 10inch rail wheels from the contact rollers through belt and pulley. Some sensors have been added to make the system autonomous. LIDAR from Velodyne has been added for 3D Map creation, RTK GNSS GPS has been added for Localization. The system is also equipped with IMU to ensure stability. Figure 2 shows the whole system in simulated environment with ROS and Gazebo simulator while Figure 3 and 4 shows the assembled system for off-track and on-track operation, respectively.

\section{Structure Investigation}

Understanding the joints type and associated link is necessary to fully enable autonomous execution of inspection and repair tasks. The arm has 6 revolute joint and their limit is from $0^{0}$ to $360^{\circ}\left(\mathrm{J}_{1}\right.$ to $\left.\mathrm{J}_{6}\right)$. Warthog UGV has total of 6 joints. Among them 4 are continuous and 2 are revolute. 4 continuous joints are situated in 4 wheels $\left(J_{9}\right.$ to $\left.J_{12}\right)$. Left side 2 wheels are connected and driven with one motor. This is the same for the right-side motors. Both the pairs have revolute joints with respect to chassis and have the limit of $-45^{0}$ to $+45^{\circ}\left(\mathrm{J}_{7}\right.$ and

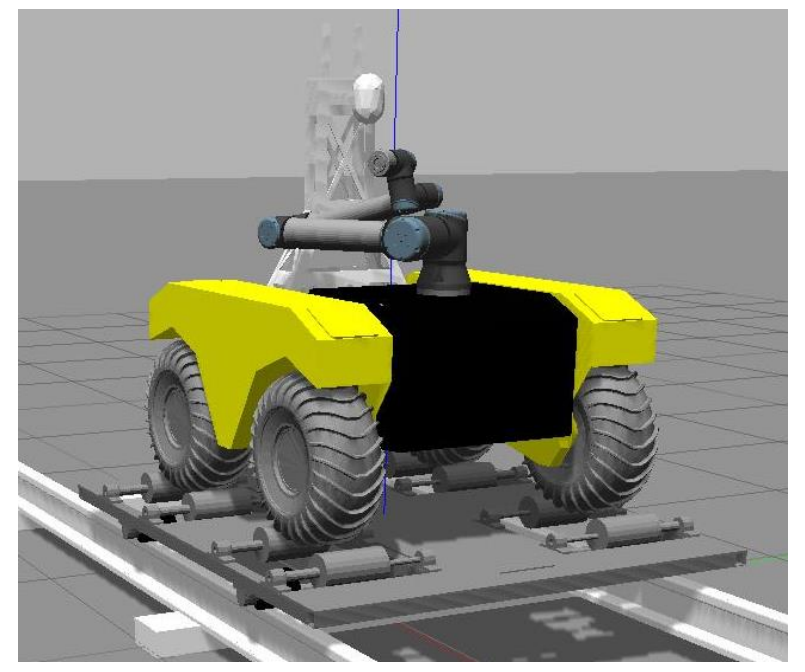

Fig. 2. Proposed RIRS in gazebo simulation

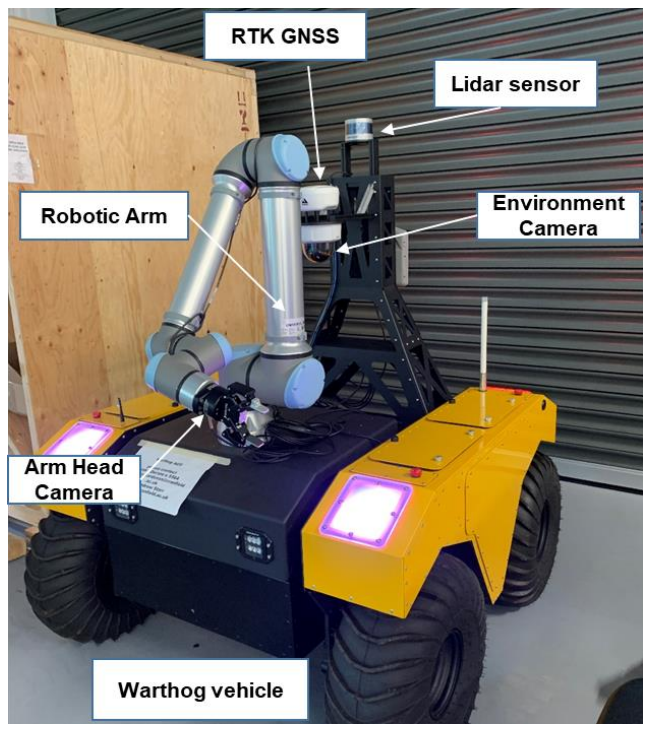

Fig. 3. Proposed RIRS for off-track inspection

$\mathbf{J}_{8}$ ). These two joints help the UGV to move upward or downward in any type of terrain. For the trolley, 2 contact rollers will contact each UGV wheel and pair of contact rollers are considered as a single joint for simplification. There are total of 4 pairs of contact rollers. Finally, four 10inch rail wheel has 4 continuous joints.

\section{1) On-track operation}

Due to the difference of dynamic motion of the vehicle between the on-track and off-track scenario, the number of joins and performance will vary depending on on-track and off-track operation. When the RIRS is engaged in on-track operation, it has total of 20 joints. The railway track will guide the RIRS and all 4 wheels will run at the same speed. There will be no left or right turn initiated from the RIRS. In this scenario, the following simplification can be considered:

a. A combination of one warthog wheel, one pair of contact roller, and one 10inch rail wheel can be considered as a single joint. For example, $\mathbf{J}_{9}, \mathbf{J}_{13}$ and $\mathbf{J}_{17}$ are acting as a single joint. This simplification will

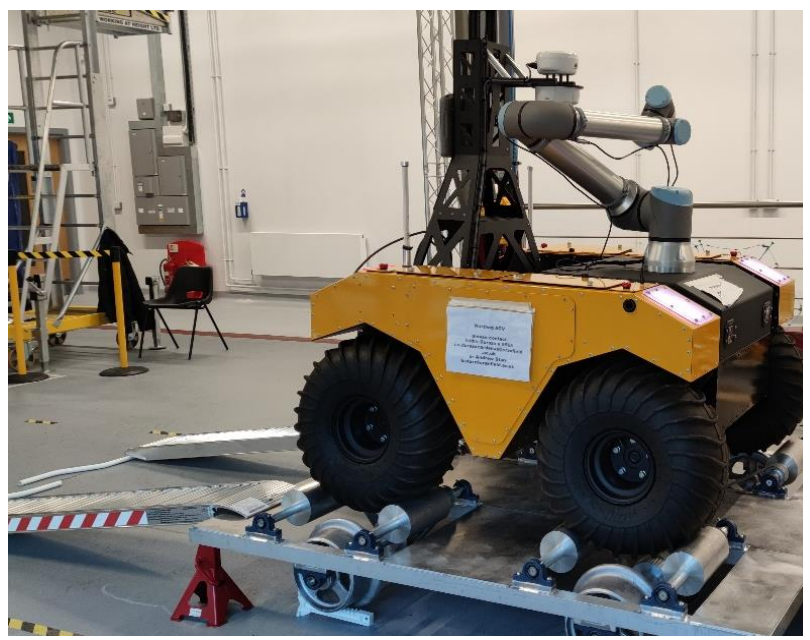

Fig. 4. Proposed RIRS for on-track inspection and repair 


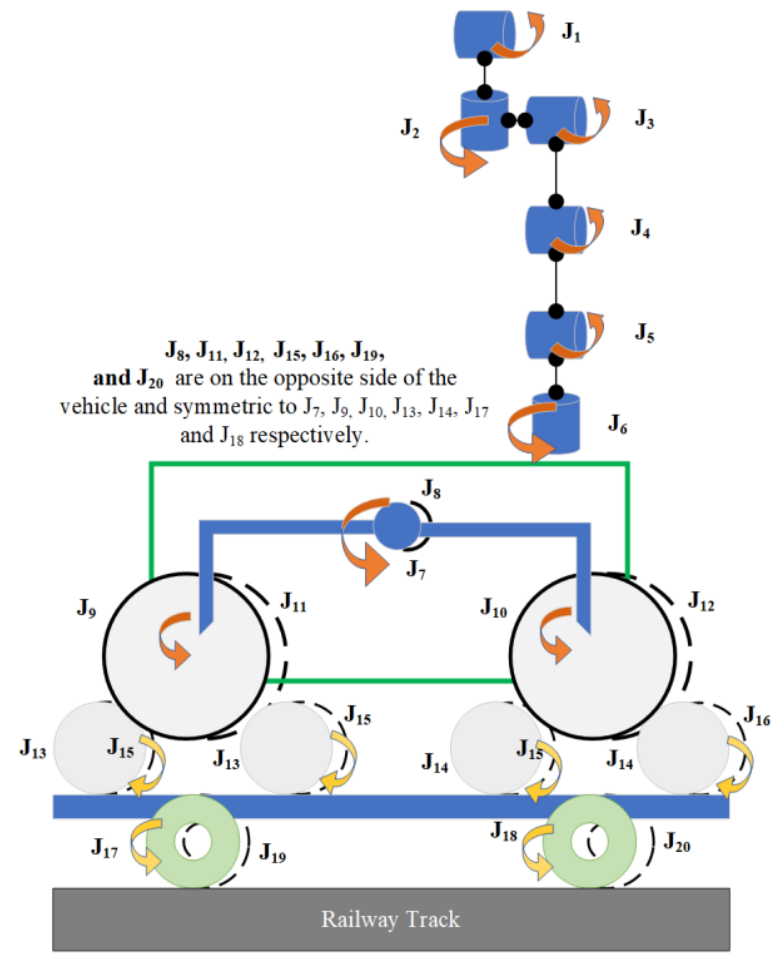

(a)

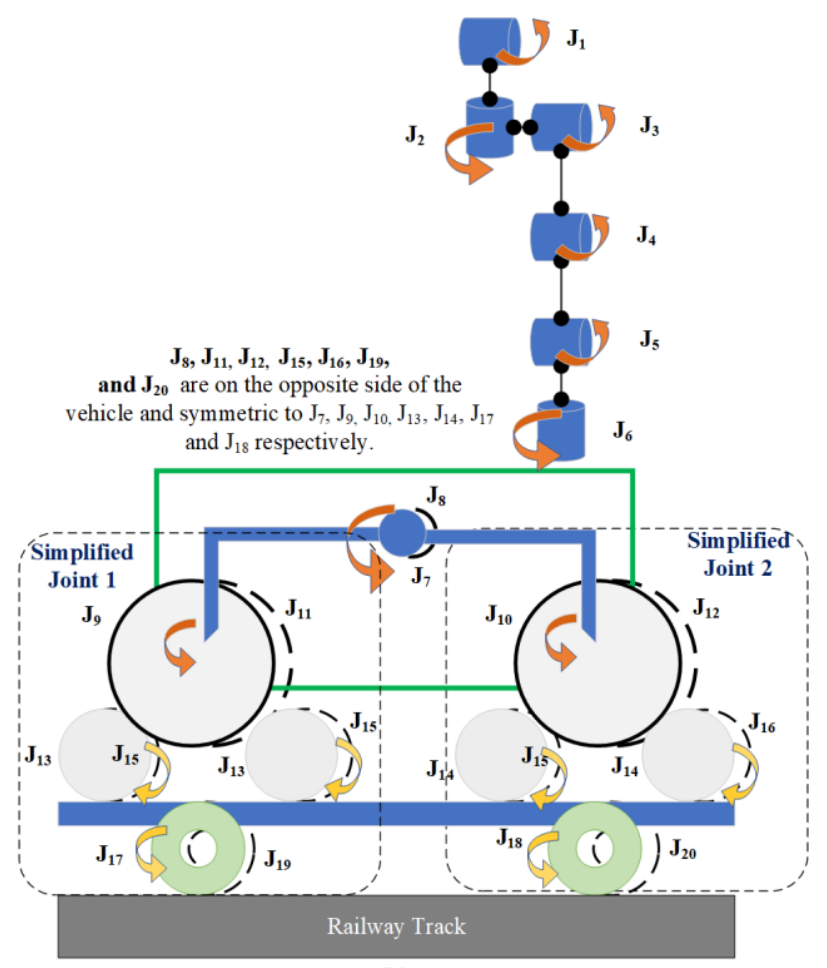

(b)

Fig. 5. Joints of RIRS for on-track operation (a) total joint (b) after simplification

reduce the number of joints and eliminate some complexity in programming.

b. As the track can be considered as a flat surface, both the revolute joint in the warthog $\left(\mathrm{J}_{7}\right.$ and $\left.\mathrm{J}_{8}\right)$ will be useless. Hence, can be eliminated.

After simplification, we can consider a prismatic joint on the lower part of the mobile manipulator where the railway track is

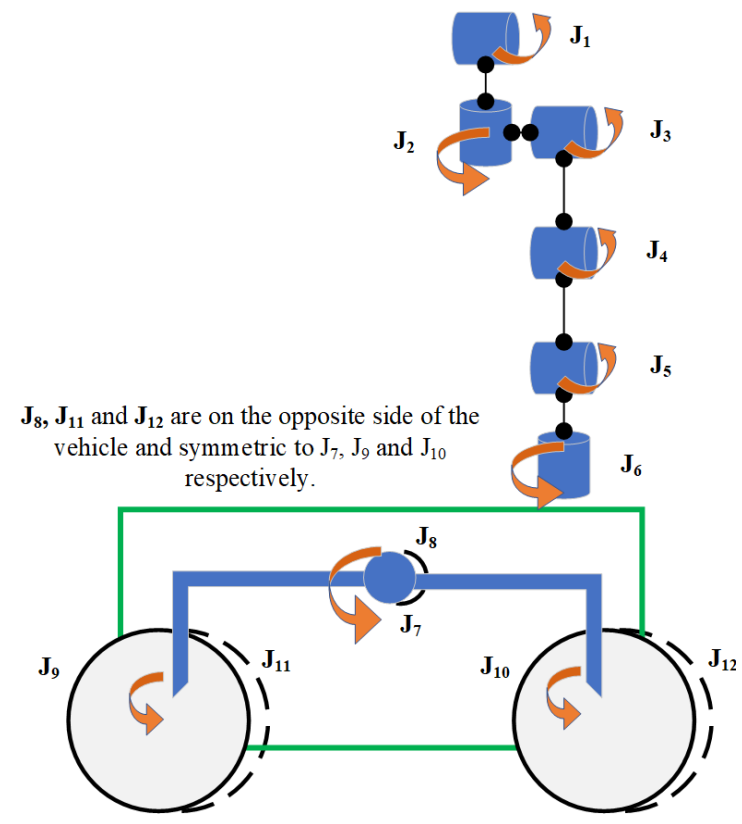

Fig. 6. Joints of RIRS for off-track operation acting as a guide. Hence, the number of joints has been reduced to 7 from 20. Figure 5 shows the joint analysis during on-track operation.

\section{2) Off-track operation}

When the RIRS is navigating off-track, then the trolley is not required anymore. So, in this scenario, no need to consider the trolley joints. There will be total of 12 joint: 6 joints of the manipulator and 6 joints of the warthog. Figure 6 shows the joints during off-track operation.

\section{DISCUSSION}

This research is intended to provide an autonomous solution for railway track (on-track and off-track) inspection and repair with the help of RIRS. By integrating a manipulator with an UGV, a multi-purpose mobile manipulator has been created for demonstration of the RIRS architecture principles. The functionality of the mobile manipulator has been extended for using it for on-track operation by using a simple trolley. Though the velocity loss among the warthog UGV wheel and contact rollers of the trolley is yet to be find out. The initial design, earlystage simulation and testing, a framework for autonomy for the RIRS has been completed.

As the structure and number of joints can be reconfigured based on on-track and off-track operation, structural investigation and joints simplification is important for next stage programming to ensure optimal trajectory and obstacle avoidance. On-track operation and off-track operation have different types of complexity such as the vibration will be different in on-track than the off-track. Also, the arm position will be different in on-track and off-track operation. In on-track 
operation, arm will be operated only in forward or backward of the UGV while during off-track operation arm can be positioned on the side of the UGV.

On-track and off-track operational capability has made this proposed RIRS unique and provides many advantages over the conventional RIRS. This is an ongoing research and in the coming days, research will be continued to integrate and improve vision system for on-track and off-track inspection, find out control system for the manipulator. At the later stage of this research, control and command system for an autonomous RIRS will be developed and tested to improve the TRL of an autonomous RIRS system.

\section{CONCLUSION}

In conclusion, the proposed RIRS system has been designed to perform both on-track and off-track operations which can be extensively used in railway track maintenance. Road Rail Conversion technique can be used with a different mobile manipulator which enables easy replication capability. Structural investigation and simplification of joints have paved the path for programming to achieve autonomy. Successful completion of this research will provide the proof of concept of using a robot with commercial rail inspection equipment.

\section{ACKNOWLEDGMENT}

This research is funded by Network Rail. This project is a part of collaboration between The IN2SMART2 project and IN2TRACK2.

\section{REFERENCES}

[1] 'A Short Guide to Network Rail - National Audit Office (NAO) Report', National Audit Office. https://www.nao.org.uk/report/a-shortguide-to-network-rail/ (accessed Jun. 16, 2020).

[2] 'New Measurement Train (NMT) - Network Rail'. https://www.networkrail.co.uk/running-the-railway/looking-after-therailway/our-fleet-machines-and-vehicles/new-measurement-train-nmt/ (accessed Jun. 17, 2020).

[3] 'Doctor Yellow', Wikipedia. May 04, 2020, Accessed: Jun. 17, 2020. [Online]. Available: https://en.wikipedia.org/w/index.php?title=Doctor_Yellow\&oldid=9547 75978.
[4] 'China Railway comprehensive inspection trains', Wikipedia. Apr. 26, 2020, Accessed: Jun. 17, 2020. [Online]. Available: https://en.wikipedia.org/w/index.php?title=China_Railway_comprehens ive_inspection_trains\&oldid $=953328584$.

[5] R. K. W. Vithanage, C. S. Harrison, and A. K. M. M. DeSilva, 'Enhance 3D Point Cloud Accuracy Through Supervised Machine Learning for Automated Rolling Stock Maintenance: A Railway Sector Case Study', in 2018 International Conference on Computing, Electronics Communications Engineering (iCCECE), Aug. 2018, pp. 242-246, doi: 10.1109/iCCECOME.2018.8658788.

[6] T. Tomiyama, L. R. García, A. Kršlin, and G. Taykaldiranian, 'Systems and Conceptual Design of a Train Cab Front Cleaning Robot', Procedia CIRP, vol. 59, pp. 61-66, Jan. 2017, doi: 10.1016/j.procir.2016.09.031.

[7] N. Mahfuz, O. A. Dhali, S. Ahmed, and M. Nigar, 'Autonomous railway crack detector robot for bangladesh: SCANOBOT', in 2017 IEEE Region 10 Humanitarian Technology Conference (R10-HTC), Dec. 2017, pp. 524-527, doi: 10.1109/R10-HTC.2017.8289014.

[8] J. James, J. Wilson, J. Jetto, A. Thomas, and V. K. Dhahabiya, 'Intelligent track cleaning robot', in 2016 IEEE International Conference on Mechatronics and Automation, Aug. 2016, pp. 332-337, doi: 10.1109/ICMA.2016.7558584.

[9] J. Dhiyaneswaran, M. Vimal raja, B. Ashok kumar, and C. B. Muthu kumaran, 'Design and fabrication of railway track cleaning bot', Mater. Today Proc., Oct. 2020, doi: 10.1016/j.matpr.2020.08.524.

[10] Karthik Rao Kordale, Shilpa Surroch, Noxious D, and more, Nemo rail robotic inspection and repair demo_3D printing. 2019.

[11] F. Comino, 'COBOTS FOR MAINTENANCE IN THE RAILWAY INDUSTRY', Thesis, Cranfield University, UK, 2018.

[12] E. Menendez, J. G. Victores, R. Montero, S. Martínez, and C. Balaguer, 'Tunnel structural inspection and assessment using an autonomous robotic system', Autom. Constr., vol. 87, pp. 117-126, Mar. 2018, doi: 10.1016/j.autcon.2017.12.001

[13] A. Kitamura, T. Namekawa, K. Hiramatsu, and Y. Sankai, 'Operating Manipulator Arm by Robot Suit HAL for Remote In-Cell Equipment Maintenance', Nucl. Technol., vol. 184, no. 3, pp. 310-319, Dec. 2013 , doi: 10.13182/NT13-A24988.

[14] S. D'Errico, 'SIMULATION OF A RAILWAY ASSET MAINTENANCE ROBOT', Cranfield University, 2018.

[15] 'Autonomous inspection trolley for better train track maintenance | Result in Brief | H2020', CORDIS | European Commission. https://cordis.europa.eu/article/id/250858-autonomous-inspectiontrolley-for-better-train-track-maintenance (accessed Dec. 26, 2020).

[16] H. Rowshandel, 'The development of an autonomous robotic inspection system to detect and characterise rolling contact fatigue cracks in railway track', p. 195.

[17] 'Our Platform - RailPod, Inc.' https://rail-pod.com/?page_id=2527 (accessed Dec. 25, 2020). 
2021-04-02

\section{Towards an autonomous RIRS: design, structure investigation and framework}

Rahman, Miftahur

IEEE

Rahman M, Liu H, Durazo Cardenas I, et al., (2021) Towards an autonomous RIRS: design, structure investigation and framework. In: IEEE 7th International Conference on Mechatronics and Robotics Engineering, 2021 (ICMRE), 3-5 February 2021, Budapest, Hungary https://doi.org/10.1109/ICMRE51691.2021.9384846

Downloaded from Cranfield Library Services E-Repository 